\title{
POTENTIAL USE OF eRHIC's 10-TO-20 GEV ERL FOR FELS AND LIGHT SOURCES
}

\author{
Vladimir N. Litvinenko and Ilan Ben-Zvi \\ Brookhaven National Laboratory, Upton, NY, USA
}

\begin{abstract}
* Work performed under the auspices of the U.S. Department of
Abstract
One of the designs of a future electron-hadron
collider, eRHIC, is based on a 5-10 GeV high current energy-recovery linac (ERL) with possible extension of its energy to $20 \mathrm{GeV}$. This ERL will operate with high brightness electron beams, which naturally match requirements for X-ray FELs and other next generation light sources. In this paper we discuss possible scenarios of using the eRHIC ERL in parasitic and dedicated mode for FELs.
\end{abstract}

\section{INTRODUCTION}

A Twenty-Year Outlook DoE document on "Facilities for the Future Science" [1] includes a new electron-hadron collider at BNL, based on the Relativistic Heavy Ion Collider (RHIC) and a new electron accelerator. The main goal of the eRHIC is to explore the physics at so-called "low-x", and the physics of color-glass condensate in electron-hadron collisions [2]. In response, the Collider-Accelerator Department at BNL in collaboration with Bates Laboratory at MIT issues the eRHIC ZDR $\left(0^{\text {th }}-\right.$ order design report), which includes a linac-ring eRHIC design based on $10 \mathrm{GeV}$ ERL [3]. The design is based on $\mathrm{CW}$ linac with high current super-conducting RF (SRF) 5-cell cavities, which are under construction at BNL [4,5] and a polarized photo-injector using a dedicated $2 \mathrm{~kW}$ circularly polarized FEL [3]. The main parameters of the eRHIC can found elsewhere [12]. The parameters are very impressive and can easily be used as a next generation light source.

In addition, RHIC rings operate only for about 30 weeks per year, which leaves about 4 and a half months for dedicated mode of ERL operation as a light source. In this case we suggest using a lower charge per bunch and a higher rep-rate, while keeping the same level of average e-beam current $(\sim 500 \mathrm{~mA})$. The e-beam parameters for this mode of operation are listed in Table 1.

Table 1. Main parameters in dedicated mode

\section{Electrons}

Beam rep-rate $[\mathrm{MHz}] \quad 703.75$

Beam energy $[\mathrm{GeV}] \quad 5-20$

$\gamma$, Relativistic factor $\quad 1-410^{4}$

RMS normalized emittance $[\mu \mathrm{m}] \quad 0.9$

Beam emittance @ $20 \mathrm{GeV}[\AA] \quad 0.18$

Full transverse coherence $\lambda[\AA]$

photon energy $[\mathrm{keV}] \quad 11$

$\begin{array}{ll}\text { RMS Bunch length [psec] } & 0.03-3 \\ \text { Charge per bunch [nC] } & 0.7 \\ \text { Average e-beam current [A] } & 0.5\end{array}$

Parameters of the electron beam in eRHIC's ERL naturally match the requirements for a next generation light source. First, let us note that the eRHIC ERL light source would have transverse coherence in the X-ray range up to $11 \mathrm{keV}$. Second, $20 \mathrm{GeV}$ operation of the ERL will extend the range of spontaneous radiation from undulators and bending magnets into $\lambda$-ray range and open new possibilities for low energy nuclear physics. Third, the ERL is a perfect driver for high power (i.e. kilowatts of $\mathrm{CW}$ power) FELs ranging from hard X-rays to VUV. Fourth, it is also a perfect match for generating beam of very intense circularly polarized $\lambda$-rays with energies up to $12 \mathrm{GeV}$.

In this paper, we take a first look at the potential of the ERL at eRHIC as a light source. We describe two possible layouts, discuss the effects of synchrotron radiation in the ERL's arcs on the e-beam parameters and, finally, present a straw-man design of X-ray FELs based on SASE, distributed optical klystron (DOK) and optics-free oscillator schemes.

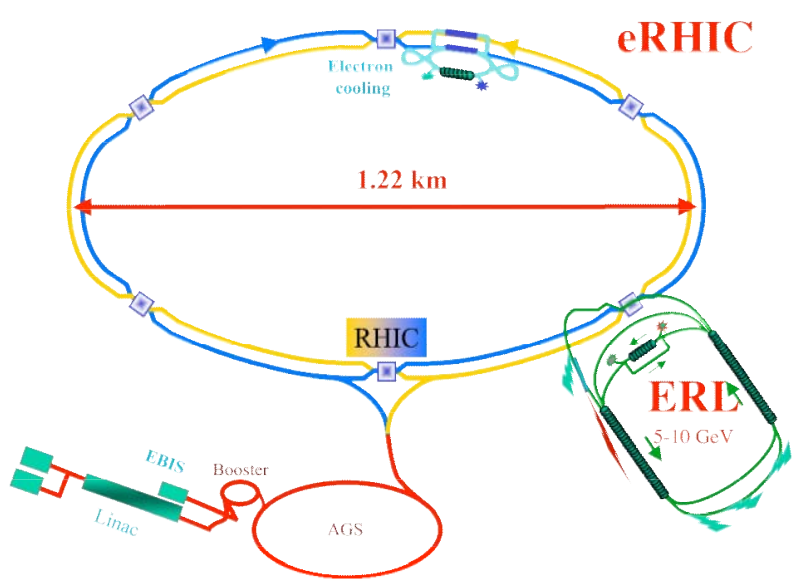

Fig. 1 eRHIC with a stand-along $10 \mathrm{GeV}$ ERL.

\section{LAYOUTS OF THE ERL-based eRHIC}

Fig.1 shows a possible layout for a stand-along ERL. The electron beam, generated in SRF photo-injector is accelerated to an energy of $0.5 \mathrm{GeV}$ by low energy ERL, followed by two passes through high energy linacs. This layout provides for a natural use of the beam-lines installed on the 2.5, 5, 7.5 and $10 \mathrm{GeV}$ arcs 
for a variety of sources of spontaneous radiation. After passing through the collision point, the beam is going through the energy recovery process, which doubles the beam current in each arc (except that at $10 \mathrm{GeV}$ ). FEL can be installed in a straight section connecting the end of the $10-\mathrm{GeV}$ arc with the linac. This scheme is limited to maximum energy of $\sim 10 \mathrm{GeV}$ by the size of the accelerator and the acceptable power of the synchrotron radiation. Fig. 2 shows a more attractive layout of ERL sharing a $3.8 \mathrm{~km}$ tunnel with two hadron storage rings (called the blue and the yellow).

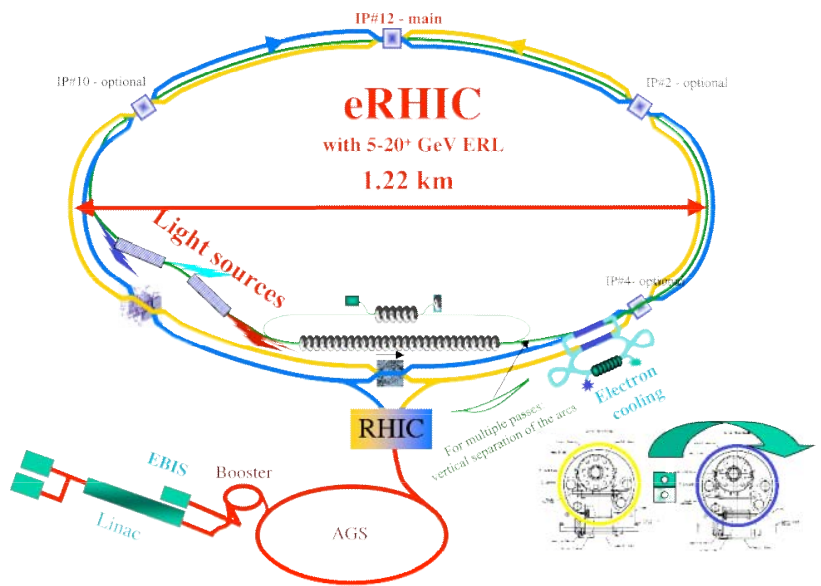

Fig. 2 Layout of the eRHIC ERL in the RHIC tunnel.

This layout provides for multiple collision points for eRHIC and also allows an increase in the energy of electron beam to $20 \mathrm{GeV}$ or above while keeping power of synchrotron radiation under control (and especially keeping linear power density well below 1 $\mathrm{kW} / \mathrm{m})$. Obviously, this layout has many advantages for the light source applications. It has best emittance and hundreds of meters of free space for hard X-ray FELs.

Similarly to other ERLs, the electron beam is generated in the gun, pre-accelerated in the low energy SRF linac, then injected into the main high energy SRF linac, which it passes twice to reach maximum energy of $20 \mathrm{GeV}$. Then the beam is decelerated to recover its energy back into RF field and is finally dumped.

Table 2. Parameters of the $20 \mathrm{GeV}$ e-beam

$\begin{array}{lcll}\text { Energy } & 20 & \mathrm{GeV} & \\ \text { Radius, average } & 610.20 & \mathrm{~m} & \\ \mathrm{~B}, \text { magnets } & 1.67 & \mathrm{kGs} & \\ \varepsilon_{\text {norm }} & 9.50 \mathrm{E}-07 & \mathrm{~m} \mathrm{rad} & \\ \varepsilon & 0.243 & \AA \mathrm{rad} & \\ \text { Bunch length } & \text { from } 0.1 \text { to } 2 & \mathrm{psec} & \\ \Delta & 0.016 & \AA \mathrm{rad} & 6.70 \% \\ \Delta, \text { final } & 0.259 & \AA \mathrm{rad} & \\ \text { RMS energy spread } & 2.54 \mathrm{E}-05 & & \end{array}$

On the way to the final energy the electron beam passes through the arcs, where synchrotron radiation may significantly affect the e-beam parameters. Our present design is based on 150 25-meter long achromatic cells with a bending radius of $400 \mathrm{~m}$ in the dipoles. The energy loss for synchrotron radiation is 35 $\mathrm{MeV}$ for $20 \mathrm{GeV}$ electrons.

The beam parameters at the end of the arc for 20 $\mathrm{GeV}$ beam and some basic parameters of the arcs are given in Table 2. In this design, synchrotron radiation determines the energy spread of both $10 \mathrm{GeV}$ and 20 $\mathrm{GeV}$, while contribution of the initial energy spread is negligible. In contrast, this low emittance lattice of the arc provides for essential preservation of the initial normalized transverse emittance: the horizontal emittance grows by $0.1 \%$ for $10 \mathrm{GeV}$ beam and by $6.7 \%$ for $20 \mathrm{GeV}$ beam. Extremely high quality of the electron beam in eRHIC's ERL provides for a natural use of its strong spontaneous radiation from its regular or specialized magnets (see Table below).

Table 3. Synchrotron radiation at $20 \mathrm{GeV}$.

$\begin{array}{lcl}\text { Loss per turn } & 35.40 & \mathrm{MeV} \\ \text { Power } & 17.70 & \mathrm{MW} \\ \lambda_{\mathrm{c}}(\text { regular bend }) & 0.28 & \AA \\ \mathrm{E}_{\mathrm{c}}(\text { regular bend }) & 44.35 & \mathrm{KeV} \\ E_{c}(10 \text { T bend }) & 0.0047 & \AA \\ E_{c}(10 \text { T bend }) & 2661 & \mathrm{KeV}\end{array}$

Energy of $20 \mathrm{GeV}$ makes building hard X-ray sources an easy task: a wiggler with period of $2 \mathrm{~cm}$ will generate fundamental wavelength of $\sim 0.1 \AA$. The range of expected spectral brightness for these sources of spontaneous radiation.

\section{ERL-based FELs}

Table 4. Main parameters of X-ray FEL driven by ERL at eRHIC

\begin{tabular}{lcccc}
\hline Energy, GeV & 20 & 20 & 10 & 10 \\
\hline Wavelength, § & 0.5 & 1 & 2 & 4 \\
Bunch length, psec & 0.2 & 0.2 & 0.4 & 0.4 \\
Peak Current, kA & 5 & 5 & 2.5 & 2.5 \\
Wiggler period, cm & 2.5 & 3 & 2.5 & 3 \\
SASE gain length, m & 7.5 & 4.3 & 3.7 & 2.4 \\
SASE Saturation length, m & 100 & 60 & 51 & 34 \\
Saturation power, GW & 7.7 & 19 & 4.5 & 9 \\
DOK, gain length, m & 3.5 & 1.4 & 0.51 & 0.25 \\
DOK, saturation length, m & 47 & 19 & 7 & 3.5 \\
\hline
\end{tabular}


Low emittance, high peak current of the $20 \mathrm{GeV}$ ERL operating in dedicated mode (Table 1) make it an attractive driver for high gain X-ray FELs, which can operate in SASE [6], and HGHG [7] modes. The very low energy spread of this source makes it attractive to use a scheme of distributed optical klystron (DOK) [8]. DOK reduces the gain length X-ray FELs at eRHIC 2.2 and 5 fold at energies of $20 \mathrm{GeV}$ and $10 \mathrm{GeV}$, correspondingly [12]. Table 4 summarizes the parameters of ERL based FELs at eRHIC. A simple use of SASE FEL combined with a very high repetition rate of ERL at eRHIC leads to extremely high average lasing power from 0.6 MW to $1.3 \mathrm{MW}$ at wavelength

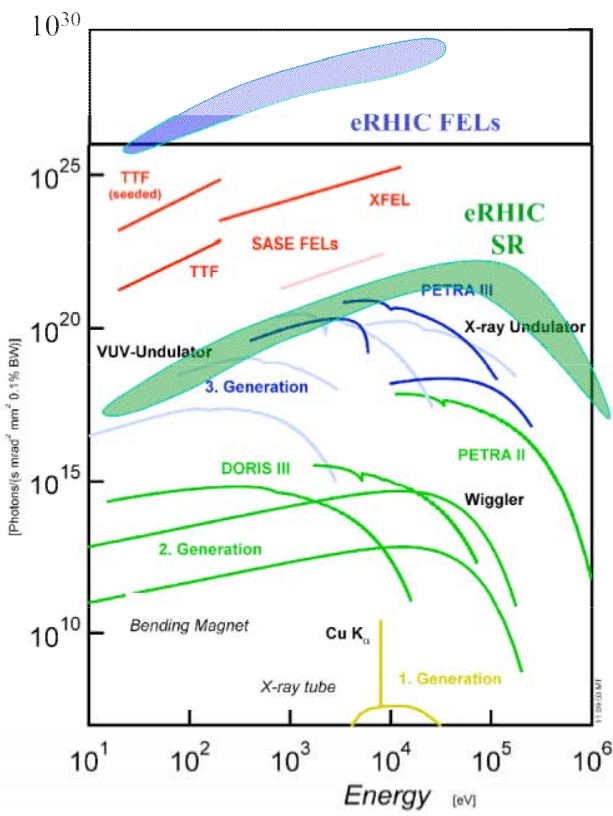

of $1 \AA$ (photon energy $12 \mathrm{keV}$ ). The solution for this potential problem is either to use Fourier limited source. i.e. either HGHG FEL or optics-free FEL oscillator (OFFO) [10,12]. The advantage of this scheme is that this FEL is fully tunable, optics independent and has a line-width of an oscillator. This scheme would allow reducing the line-width of X-ray FEL to a few parts per million compared with a few parts per thousands, typical for SASE FELs [11]. In this case, the average power of X-ray FEL at eRHIC with average spectral brightness in $10^{30}$ range (see Fig.3) will reduce to manageable levels of tens of hundreds of watts.

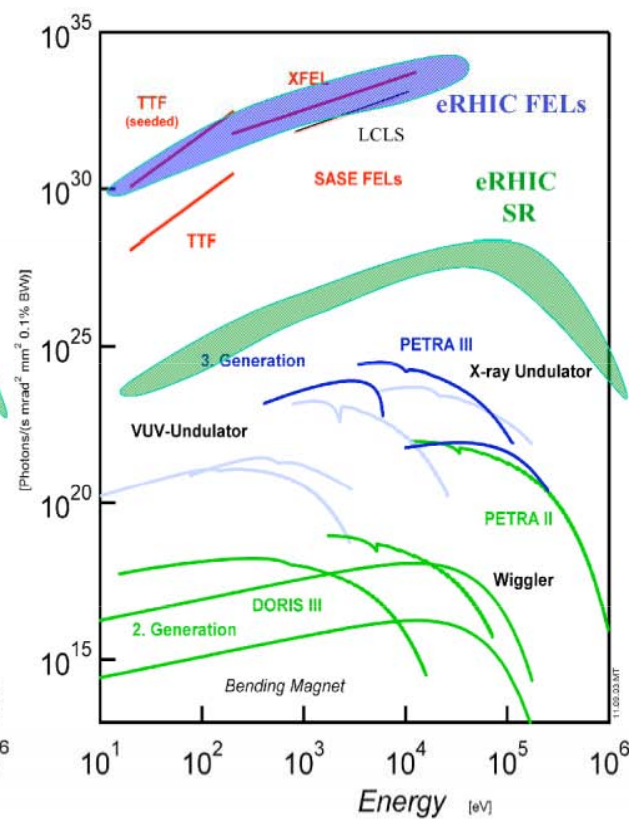

Fig.3 Average and peak spectral brightness of eRHIC light source (blue - FELs, green - spontaneous radiation) compared with other sources. While being similar to SASE FEL sources in peak spectral brightness, the average spectral brightness of eRHIC FELs would exceed the rest of the light sources (existing and proposed) by about 5 orders of magnitude.

\section{CONCLUSION}

Future high current 10-20 GeV ERL for eRHIC electron-hadron collider has outstanding potential to become a next generation light source with unprecedented levels of average spectral brightness.

This work is supported by the US Department of Energy and Office of Naval Research.

\section{REFERENCES}

[1] http://www.er.doe.gov/Sub/Facilities for future/facilities future.htm

[2] http://www.bnl.gov/henp/docs/NSAC_RHICII-eRHIC_2$15-03$.

[3] http://www.agsrhichome.bnl.gov/eRHIC/, Appendix A, Linac-Ring Option

[4] I. Ben-Zvi at al., Ampere Average Current Photo-Injector and Energy Recovery Linac, these Proceedings.
[5] R. Calaga et al., High Current Superconducting Cavities at RHIC, Proceedings of EPAC-2004, Geneva, Switzerland, July 5-9, 2004

[6] A.M. Kondratenko, and E.L. Saldin, Particle Accelerators 10, 207 (1980); J.B. Murphy and C. Pellegrini, Nuclear Instruments, and Methods A237, 159 (1985);

[7] L.H. Yu et al, Physics Review Letters 91, No. 7, 0748011 (2003)

[8] V.N. Litvinenko, Nuclear Instruments and Methods A 304, (1991) 463

[9] N.A. Vinokurov and A.N . Preprint INP 77-59, Novosibirsk, 1977

[10] V.N. Litvinenko, Proceedings of FEL conference, August 2002, APS, Argonne, IL, Eds. K.-J.Kim, S.V.Milton, E.Gluskin, p. II-1

[11] V. Ayvazyan et al., Physics Review Letters 88, 104802 (2002)

[12] V.N. Litvinenko and I. Ben-Zvi , Proceedings of FEL'2004 Conference, August 30 - September 3, 200e, Trieste, Italy, pp. 594-597, http://accelconf.web.cern.ch/AccelConf/f04/ 